ing from the $N$ th mode in a point contact have $N$ maxima in $|\Psi|^{2}$ (where $\Psi$ is the wavefunction).

In the full quantum mechanical calculations of the pattern of electron flow from the lowest three modes of a QPC (Fig. 4, D to F), the color scale represents the wavefunction $|\Psi|^{2}$ of electrons [Figs. 4D (first mode), 4E (second mode), and 4F (third mode)]. The wavefunction patterns agree well with images of electron flow; in both, the angular pattern of electrons flowing from the $N$ th mode has $N$ lobes. For these calculations, the transverse potential of the QPC was parabolic, with varying curvature to produce an electron gas channel approximating the lithographic shape of the gates; the white curves in Fig. 4, D to F, show where the potential crosses the Fermi energy. To ease comparison, areas not scanned in the experiment are dimmed in the simulation.

Figure 4, G to I, plots the angular distribution of measured electron flow in Fig. 4, A to $\mathrm{C}$. The circular slice used to determine the distribution is shown in Fig. 4A. Figure 4, J to L, plots the angular distribution of electron wavefunctions $|\Psi|^{2}$ from Fig. 4, D to F, taken using a circular slice shown in Fig. 4D. Comparison shows a high degree of agreement between theory and experiment. The measured angular dependencies of images and theory have the same modal structure ( $N$ lobes for the $N$ th transverse mode), and they agree quite well in shape.

The height of the $N$ th conductance plateau is changed when the AFM tip is held at a fixed position above the electron flow pattern from the $N$ th mode of the QPC (Fig. 5). When no tip is present (Fig. 5A), each transverse mode contributes $2 e^{2} / h$ of conductance. When the tip is placed directly in front of the QPC (Fig. 5B), it backscatters electrons from the first mode without affecting electrons from the second (Fig. 4D). This reduces the entire first plateau to $1.8 e^{2} / h$ without changing the spacing between the first and second plateaus. When the tip is placed off-axis as in Fig. 5C, it backscatters electrons from the second mode without affecting electrons from the first (Fig. 4E). This reduces the spacing between the first and second plateaus to $1.7 e^{2} / h$ without affecting the height of the first plateau.

We have presented spatial images that show the coherent flow of electron waves through semiconductor nanostructures. The observation of the expected angular structure of electron flow from the first, second, and third modes of a QPC, as well as the clear appearance of fringes separated by half the Fermi wavelength $\lambda_{\mathrm{F}} / 2$, are strong confirmations of this imaging technique. These results are a confirmation of the power that SPMs have for imaging the flow of electron waves through mesoscopic semiconductor devices. The ability to image elec- tron wave flow inside GaAs/AlGaAs heterostructures makes possible future experiments, including spatial investigations of quantum coherence, universal conductance fluctuations, and weak localization.

\section{References and Notes}

1. M. F. Crommie, C. P. Lutz, D. M. Eigler, Science 262, 218 (1993).

2. E. J. Heller, M. F. Crommie, C. P. Lutz, D. M. Eigler, Nature 369, 464 (1994).

3. H. C. Manoharan, C. P. Lutz, D. M. Eigler, Nature 403, 512 (2000).

4. M. A. Eriksson et al., Appl. Phys. Lett. 69, 671 (1996).

5. R. Crook, C. G. Smith, C. H. W. Barnes, M. Y. Simmons, D. A. Ritchie, J. Phys. Cond. Mat. 12, L167 (2000).

6. M. J. Yoo et al., Science 276, 579 (1997).

7. K. L. McCormick et al., Phys. Rev. B 59, 4654 (1999).

8. N. B. Zhitenev et al., Nature 404, 473 (2000).

9. G. Finkelstein, P. I. Glicofridis, R. C. Ashoori, M. Shayegan, Science 289, 90 (2000).

10. L. Gurevich, L. Canali, L. P. Kouwenhoven, Appl. Phys. Lett. 76, 384 (2000).

11. B. J. van Wees et al., Phys. Rev. Lett. 60, 848 (1988).

12. D. A. Wharam et al., J. Phys. C Solid State Phys. 21, 209 (1988).

13. Reviewed in C. W. J. Beenakker and H. van Houten, Solid State Phys. 44, 1 (1991).

14. L. W. Molenkamp et al., Phys. Rev. B 41, 1274 (1990).
15. K. L. Shepard, M. L. Roukes, B. P. van der Gaag, Phys. Rev. Lett. 68, 2660 (1992).

16. The tips used were piezoresistive cantilevers from ThermoMicroscopes, Sunnyvale, CA; see also M. Tortonese, R. C. Barret, C. F. Quate, Appl. Phys. Lett. 62 834 (1993).

17. D. P. DiVincenzo, in Mesoscopic Electron Transport, L. L. Sohn, L. P. Kouwenhoven, G. Schön, Eds. (Kluwer Academic, Boston, MA, 1997), pp. 657-677.

18. The average spacing of these fringes is $22 \pm 2 \mathrm{~nm}$, and the expected spacing from the sheet density is $\lambda_{\mathrm{F}} / 2=19 \mathrm{~nm}$. The difference can be attributed to a lower sheet density in the area of the device as compared with the bulk, because of the negatively charged gates and AFM tip.

19. B. L. Altshuler, A. G. Aronov, D. E. Khmelnitsky, J. Phys. C 15, 7367 (1982).

20. Supported in part at Harvard University by Office of Naval Research/Augmentation Awards for Science and Engineering Research Training (ONR/AASERT) grant N00014-97-1-0770, by ONR grant N0001495-1-0104, and by NSF under grant NSFCHE9610501 and Harvard's Materials Research Science and Engineering Center grant DMR-98-09363. M.A.T. and S.E.J.S. were supported by NSF graduate fellowships. This work was supported at the University of California, Santa Barbara, by NSF Science and Technology Center QUEST.

1 June 2000; accepted 22 August 2000

\title{
Technique for Enhanced Rare Earth Separation
}

\section{Tetsuya Uda, ${ }^{1 *} \mathrm{~K}$. Thomas Jacob, ${ }^{2}$ Masahiro Hirasawa ${ }^{1}$}

\begin{abstract}
A process is demonstrated for the efficient separation of rare earth elements, using a combination of selective reduction and vacuum distillation of halides. The large differences in the redox chemistry of the rare earth elements and in the vapor pressures of rare earth di- and trihalides are exploited for separation. Experimental proof of concept is provided for the binary systems praseodymium-neodymium and neodymium-samarium. This process enhances the separation factor for the isolation of samarium and neodymium from their mixture by more than an order of magnitude.
\end{abstract}

Rare earth elements and compounds find application in many advanced materials of current interest such as high-performance magnets, fluorescent materials, chemical sensors, high-temperature superconductors, magnetooptical disks, and nickel-metal hydride batteries. Powerful rare earth permanent magnets such as $\mathrm{Nd}_{2} \mathrm{Fe}_{14} \mathrm{~B}$ and $\mathrm{SmCo}_{5} / \mathrm{Sm}_{2} \mathrm{Co}_{17}$ have revolutionized technology, allowing miniaturization of devices such as the hard disk drive and compact disc player. However, the production cost of rare earth permanent magnets is very high, because of the high cost of extracting pure $\mathrm{Sm}$ or Nd metal used in their manufacture. The separation of individ-

${ }^{1}$ Research Center for Metallurgical Process Engineering, Institute for Advanced Materials Processing, Tohoku University, Sendai 980-8577, Japan. ²Department of Metallurgy, Indian Institute of Science, Bangalore 560 012, India.

*To whom correspondence should be addressed. Email: uda@iamp.tohoku.ac.jp ual rare earth elements is a difficult process involving solvent extraction or ion exchange (1-3). Because the chemical properties of rare earth ions in aqueous solution exhibit only incremental variation with atomic number, solvent extraction must be repeated many times. The ion exchange process is not suitable for industrial production because of the very long periods (greater than 10 days) required to accomplish significant separation. A dry process was recently developed for the separation of rare earth elements $(4,5)$ using chemical vapor transport mediated by a gaseous complex such as $\mathrm{LnAlCl}_{3}$ (Ln: lanthanoid). However, the separation efficiency with the dry process for adjacent elements in the periodic table was lower than that obtained in the conventional solvent extraction process (6). We present a more efficient separation method, which combines selective reduction with vacuum distillation.

Although rare earth elements are usually present as trivalent ions in their compounds, 


\section{REPOR T S}

many rare earth dichlorides have been synthesized. The dichlorides of $\mathrm{Nd}, \mathrm{Sm}, \mathrm{Eu}, \mathrm{Dy}$, $\mathrm{Tm}$, and $\mathrm{Yb}$ are known to be stable $(7,8)$. The molten salt containing significant amounts of divalent ions exist at high temperature for the other rare earths (e.g., Sc, La, Ce, Pr) (9). The vapor pressure of rare earth dihalides is lower than that of the corresponding trihalides by one to two orders of magnitude (10-12). Thus, preferential reduction of a component in a mixture of rare earth trichlorides can markedly affect its volatility and provide a method for its separation.

The chemical potentials of chlorine corresponding to $\mathrm{Ln}+\mathrm{LnCl}_{2}$ and $\mathrm{LnCl}_{2}+\mathrm{LnCl}_{3}$ equilibria at $1073 \mathrm{~K}$ are shown in Fig. 1. The Gibbs energies of formation of the trichlorides $\left(\mathrm{LnCl}_{3}\right)$ that are available in the literature (10) are combined with those for the dichlorides $\left(\mathrm{LnCl}_{2}\right)$, which are estimated from their enthalpies of formation $(13,14)$. For unstable $\mathrm{LnCl}_{2}$ compounds, the enthalpies of formation evaluated by Novikov and Polyachenok (15) and Johnson (16) using the Born-Haber cycle were used. These data, associated with larger uncertainty, are represented by dotted symbols in Fig. 1. When pure $\mathrm{LnCl}_{2}$ is stable, the chemical potential of chlorine corresponding to $\mathrm{Ln}+\mathrm{LnCl}_{2}$ equilibrium is more negative than that for the $\mathrm{LnCl}_{2}+\mathrm{LnCl}_{3}$ equilibrium. This is the case for the elements $\mathrm{Nd}, \mathrm{Sm}, \mathrm{Eu}, \mathrm{Dy}, \mathrm{Ho}$, $\mathrm{Tm}$, and $\mathrm{Yb}$. For the other elements, the pure dichloride is unstable, and hence, the chemical potential for the $\mathrm{Ln}+\mathrm{LnCl}_{2}$ equilibrium is more positive than that for the $\mathrm{LnCl}_{2}+\mathrm{LnCl}_{3}$ equilibrium. In these cases, some $\mathrm{LnCl}_{2}$ can exist at reduced activity in molten salts (9). It is seen that the trend in the chemical potential of chlorine for the $\mathrm{Ln}+\mathrm{LnCl}_{2}$ equilibria is opposite to that for $\mathrm{LnCl}_{2}+\mathrm{LnCl}_{3}$ equilibria: minima in the first approximately correspond to maxima in the second and vice versa. Therefore, it is possible to selectively reduce a trivalent ion in a mixture of rare earth trichlorides to its divalent state. Thus, a separation process combining se- lective reduction with vacuum distillation is feasible. This idea is demonstrated for the binary systems Pr-Nd and Nd-Sm. The rare earth metals Nd and Sm are the most important for producing strong magnets. The combination of Pr and Nd is one of the most difficult to separate by the conventional process, requiring more than 20 repetitive extractions.

Because the chemical potential of chlorine for the $\mathrm{SmCl}_{2}+\mathrm{SmCl}_{3}$ equilibrium is higher than that for $\mathrm{NdCl}_{2}+\mathrm{NdCl}_{3}$ equilibrium, the preparation of $\mathrm{SmCl}_{2}-\mathrm{NdCl}_{3}$ mixture is thermodynamically possible at $1073 \mathrm{~K}$. Aluminum can reduce $\mathrm{SmCl}_{3}$ to $\mathrm{SmCl}_{2}$ without reducing $\mathrm{NdCl}_{3}$, as illustrated in Fig. 1

$$
\begin{aligned}
& 3 \mathrm{SmCl}_{3} \text { (mixture) } \\
& +\mathrm{Al}(\mathrm{l}) \rightarrow 3 \mathrm{SmCl}_{2} \text { (mixture) }+\mathrm{AlCl}_{3}(\mathrm{~g})
\end{aligned}
$$

The removal of $\mathrm{AlCl}_{3}$ is easy because its boiling point is $456 \mathrm{~K}$. For a system containing trichlorides of $\mathrm{Nd}$ and $\mathrm{Pr}$, a stronger reductant than $\mathrm{Al}$ is required for the selective reduction of $\mathrm{NdCl}_{3}$ in the $\mathrm{NdCl}_{3}-\mathrm{PrCl}_{3}$ mixture, because $\mathrm{NdCl}_{3}$ is more stable than $\mathrm{AlCl}_{3}$. Stoichiometric amounts of $\mathrm{Nd}$ metal can be used as the reductant for the preparation of the $\mathrm{NdCl}_{2}-\mathrm{PrCl}_{3}$ mixture

$$
\begin{aligned}
& 2 \mathrm{NdCl}_{3} \text { (mixture) } \\
& \quad+\mathrm{Nd}(\mathrm{s}) \rightarrow 3 \mathrm{NdCl}_{2} \text { (mixture) }
\end{aligned}
$$

Other rare earth metals (including misch metal), alkali metals, and alkaline earth metals can also be considered as reductants for the trichlorides.

In the schematic of the apparatus used for the combined selective reduction-vacuum distillation process (Fig. 2), two electric heaters were used to generate the desired temperature gradient. A closed-end stainless steel tube was placed horizontally inside the heater assembly. Ten short concentric graphite tubes (length: 5 $\mathrm{cm}$ ) were inserted inside the stainless steel tube.
A molybdenum boat, containing a mixture of trichlorides and a reductant, was placed at the middle of the graphite ring adjacent to the closed end of the stainless steel tube. Trichlorides (purity: 99.9\%) used as the starting material were distilled once in a quartz reaction tube before use. The trichlorides in the Sm-Nd system were mixed in an equimolar ratio. In the Pr-Nd system, $\mathrm{NdCl}_{3}, \mathrm{PrCl}_{3}$, and $\mathrm{Nd}$ metal were mixed such that the rare earth elements were in an equimolar ratio. These mixtures were then transferred into the stainless steel tube in a glove box filled with Ar gas. Selective reduction was carried out in vacuum under conditions shown in Figs. 3 and 4. The temperature of the Mo boat was then raised to 1173 or $1273 \mathrm{~K}$ for the vacuum distillation, after which the stainless steel tube was cooled to room temperature and the changes in mass of the inner graphite rings were measured in the glove box. The chlorides deposited on the rings and the residue in the Mo boat were collected. The mole ratio of rare earths in the collected salt was measured by x-ray fluorescence (XRF), and phases present in the deposit were identified by $\mathrm{X}$-ray powder diffraction analysis (XRD).

Results of selective reduction of an equimolar mixture of trichlorides of $\mathrm{Sm}$ and $\mathrm{Nd}$ by $\mathrm{Al}$ metal and subsequent vacuum distillation are shown in Fig. 3. The mass recorded at $0 \mathrm{~cm}$ is the amount of the residue in the Mo boat. There was significant selective transport of $\mathrm{NdCl}_{3}$ from the boat to the cooler graphite rings via the vapor phase. The deposition occurred on the graphite rings at a temperature of about 1000 K. Samarium-rich chlorides remained in the Mo boat, whereas $\mathrm{AlCl}_{3}$ was deposited at the exhaust port at room temperature and separated completely from the rare earth chlorides. In the solvent extraction, separation factor is usually used to quantify the separability of the metal ions. The separation factor is a ratio of elemental distribution ratios between the organic and aque-

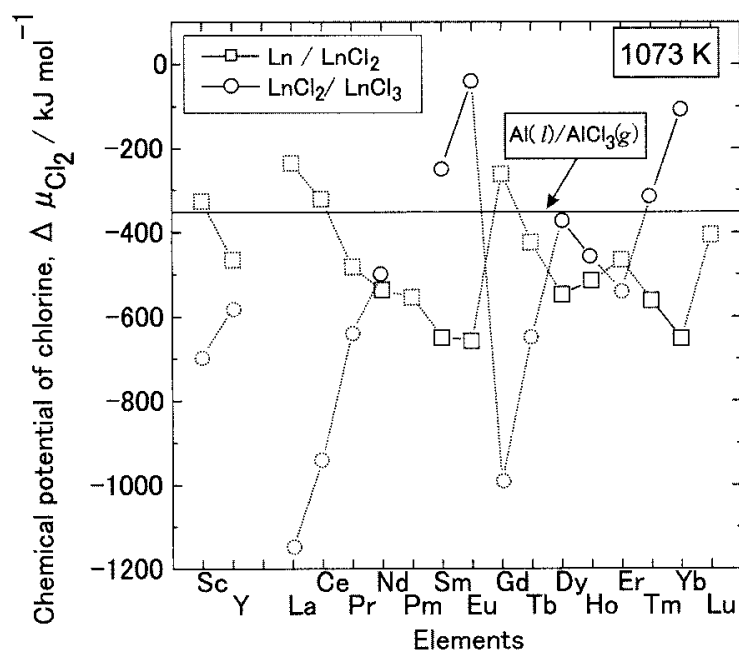

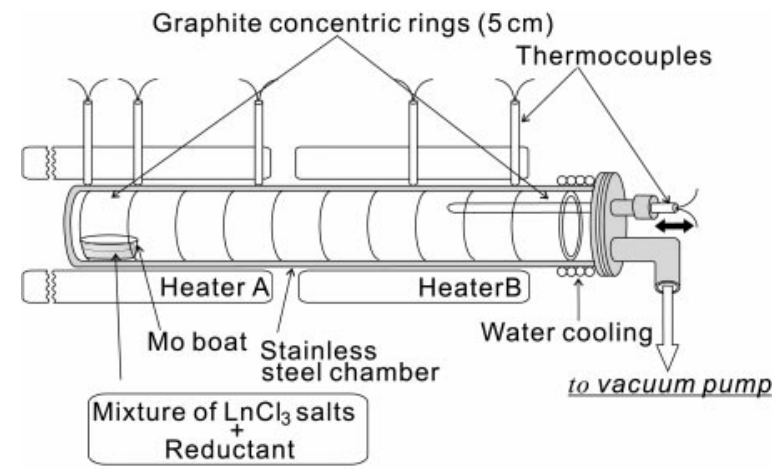

Fig. 1 (left). Chemical potentials of chlorine corresponding to the equilibria between $\mathrm{Ln}$ and $\mathrm{LnCl}_{2}$ and $\mathrm{LnCl}_{2}$ and $\mathrm{LnCl}_{3}$ at $1073 \mathrm{~K}$. Values computed from estimated data with large uncertainty are indicated by dotted symbols. Fig. 2 (right). Schematic of the apparatus for the combined selective reduction-vacuum distillation process. 


\section{R E P O R T S}

ous phases. For comparison, an analogous separation factor for the new process is defined as

$$
\beta_{\mathrm{Ln} 1 / \mathrm{Ln} 2}=\frac{\left(x_{\mathrm{Ln} 1}^{\mathrm{r}} / x_{\mathrm{Ln} 1}^{\mathrm{d}}\right)}{\left(x_{\mathrm{Ln} 2}^{\mathrm{r}} / x_{\mathrm{Ln} 2}^{\mathrm{d}}\right)}
$$

where $x_{\text {Ln }}^{\mathrm{r}}$ and $x_{\text {Ln }}^{\mathrm{d}}$ are the average mole fractions of each lanthanoid in the residue and the deposit, respectively. In the calculation of the separation factor, a very small amount of deposit collected from the graphite ring placed next to the Mo boat was added to that of the residue. The average concentrations were 91 mole percent (mol \%) Sm in the residue and $98 \mathrm{~mol} \% \mathrm{Nd}$ in the deposit. The separation factor obtained here is $\beta_{\mathrm{Sm} / \mathrm{Nd}}=\sim 570$ in contrast to the separation factor of 2.2 to 9.6 (17) reported for the conventional solvent extraction. For commercial use as components of rare earth permanent magnets, the required purity of metals is about 95 mass \% for $\mathrm{Nd}$ and 95
Fig. 3. Typical results of the combined selective reduction-vacuum distillation process using $\mathrm{Al}$ as the reductant for an equimolar mixture of $\mathrm{SmCl}_{3}$ and $\mathrm{NdCl}_{3}$. The initial mixture placed in the Mo boat contained $\mathrm{SmCl}_{3}(0.012$ $\mathrm{mol}), \mathrm{NdCl}_{3}(0.012 \mathrm{~mol})$, and excess $\mathrm{Al}(0.008 \mathrm{~mol})$.
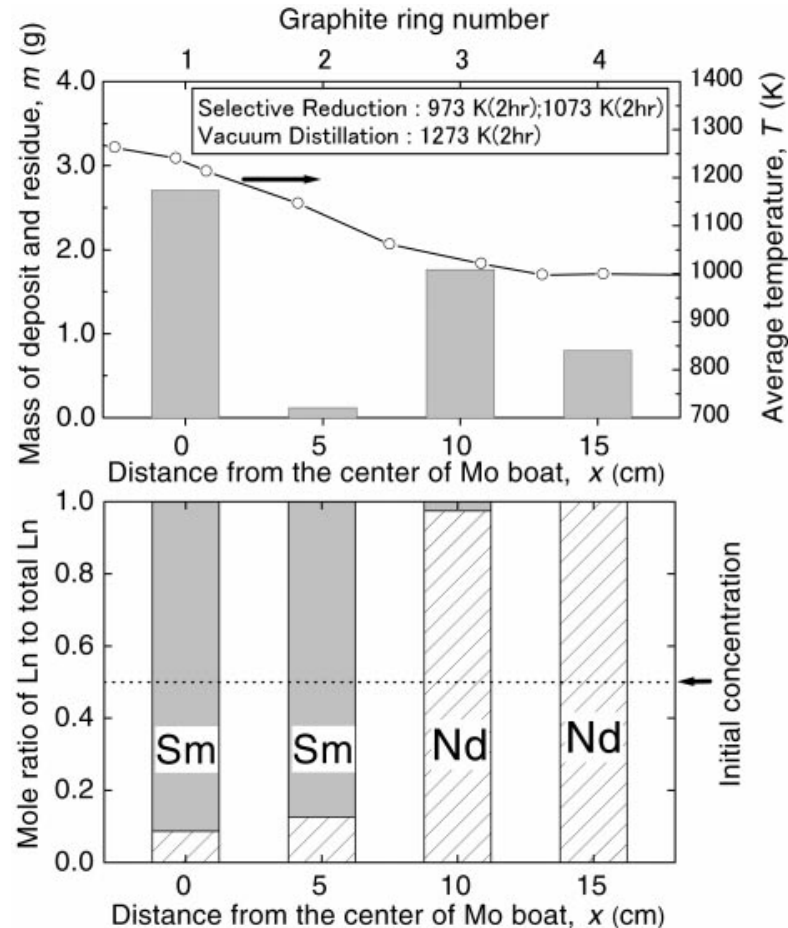

Fig. 4. Typical results of the combined selective reduction-vacuum distillation process for a mixture of $\mathrm{Nd}$ and Pr trichlorides. Neodymium metal was used as the reductant. The initial mixture placed in the Mo boat contained $\mathrm{PrCl}_{3}(0.012 \mathrm{~mol})$, $\mathrm{NdCl}_{3}(0.008 \mathrm{~mol})$, and $\mathrm{Nd}(0.004$ $\mathrm{mol})$.
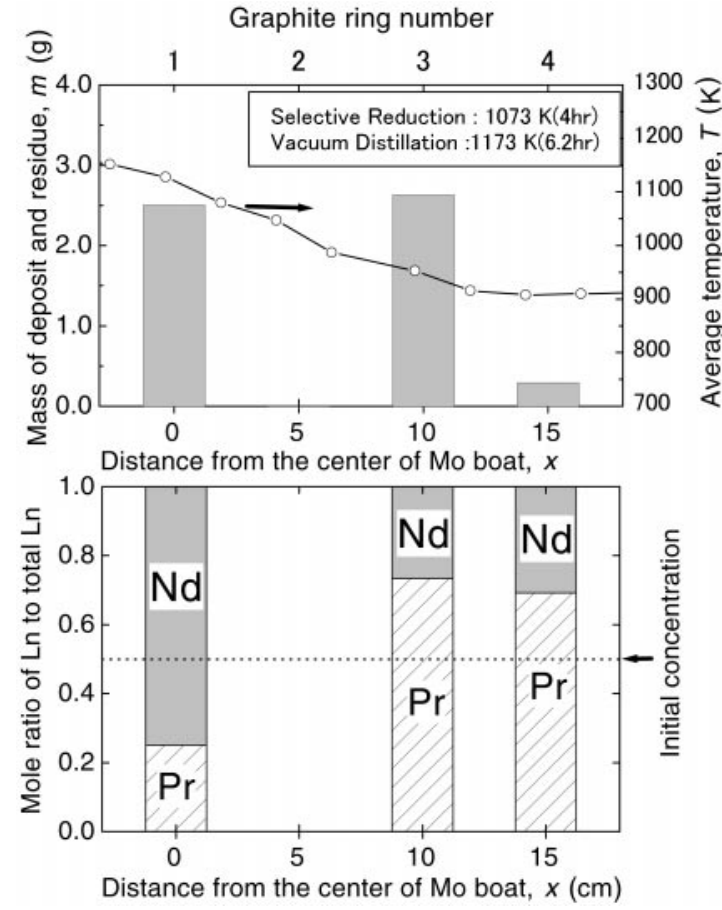

mass $\%$ for $\mathrm{Sm}$. This can be achieved in one distillation for $\mathrm{Nd}$. Preliminary experiments using lower purity trichlorides that contained a small amount of water indicated that the formation of neodymium oxychloride $(\mathrm{NdOCl})$ in the Mo boat decreases the purity of the residue because the oxychloride has a much lower vapor pressure. Thus, the residue containing more than 95\% Sm may be obtained in one distillation by completely removing oxygen impurity in the mixture.

The result of selective reduction of a mixture of $\mathrm{PrCl}_{3}$ and $\mathrm{NdCl}_{3}$ and subsequent vacuum distillation is shown in Fig. 4. The trichlorides evaporated from the boat and were deposited on the graphite rings at temperatures ranging from 900 to $1000 \mathrm{~K}$. The separation of $\mathrm{Pr}$ and Nd was less efficient than that for the Nd-Sm system. Because the difference in chemical potential of chlorine for the equilibria $\mathrm{Nd}+\mathrm{NdCl}_{2}$ and $\mathrm{NdCl}_{2}+\mathrm{NdCl}_{3}$ is small, activity of $\mathrm{NdCl}_{3}$ is fairly high. Hence, there is significant vapor pressure of $\mathrm{NdCl}_{3}$ over the chloride mixture in the boat. Further, $\mathrm{PrCl}_{3}$ may be partly reduced to $\mathrm{PrCl}_{2}$ at reduced activity in the molten salt. These factors are responsible for the less efficient separation.

The separation factor for the Pr-Nd system obtained in the new process with $\mathrm{Nd}$ as the reductant is 8.1. In contrast, the separation factor in the conventional solvent extraction using organic and aqueous media ranges from 1.3 to 1.7 (17), although a separation factor of 5.06 in one laboratory test, using a solution containing $70 \%$ acetonitrile in place of aqueous solution, was very recently reported (18). The number of cycles required to obtain the final product is proportional to $(1 / \log \beta)(17)$. Thus, when $\beta$ is small as in the Pr-Nd system, a moderate increase in the separation factor can greatly decrease the number of extractions. For example, a change in $\beta$ from 1.3 or 1.7 in the conventional process to 8.1 reduces the number of cycles to one-eighth or onefourth of those usually necessary.

By using the technique we developed, separation can be achieved not only from mixtures of rare earths, but also from mixtures of rare earths and other transition metals. Many of the transition metal chlorides such as those of $\mathrm{Fe}$ and Co have higher vapor pressures than rare earth trichlorides. A large amount of scrap containing rare earths, Fe, and $\mathrm{Co}$ is produced during the manufacture of the rare earth magnets. The process outlined here is suitable for the recovery of rare earths from this scrap.

More diiodides in the pure form are stable compared with dichlorides; there are 11 stable diiodides (Sc, La, Ce, Pr, Nd, Sm, Eu, Gd, Dy, $\mathrm{Tm}$, and $\mathrm{Yb})(19,20)$. In addition, iodides have an advantage because of their higher vapor pressure. The vaporization of iodides is expected to 
occur at relatively lower temperatures. Di- and triiodides vaporize at around $1273 \mathrm{~K}$ during vacuum distillation but condense at different temperatures. The nonvolatile impurity oxyiodides remain in the Mo boat and can be separated from the diiodides completely. Thus, the oxyiodides do not affect the separation between di- and triiodides. We have conducted preliminary experiments on the separation of $\mathrm{Sm}$ and Dy at $1273 \mathrm{~K}$ from an iodide melt. The results calculated from the compositions of diiodiderich and triiodide-rich deposits suggest a separation factor $\beta^{\prime}{ }_{\mathrm{Sm} / \mathrm{Dy}}=2300$. The value is large enough to produce pure metals. Although thermodynamic properties of diiodides at elevated temperature have not been well established, the iodide route appears to be more promising for rare earth separation using the technique described here.

Wet processes such as solvent extraction are generally associated with complicated flow sheets (1). First, the rare earth raw minerals have to be decomposed and dissolved in aqueous solution. After an elaborate separation process for rare earths involving several cycles, concentration of the solution, precipitation, filtering, drying, and calcination are required for obtaining purified rare earth oxides. In the conventional reduction processes, the oxides are directly reduced to metals or converted to halides. The halides are then reduced to metals. Thus, the halides separated by the present process can be directly transferred to the conventional halide reduction process or used in the oxide reduction process after the pyrometallurgical conversion to oxides. The dry process proposed here provides an easier path with fewer process steps than the wet process. The differences in the redox potential and vapor pressure of compounds in different oxidation states of rare earths provide better opportunities for the design of suitable separation processes compared with the conventional process using only incremental variation of chemical properties with atomic number for a given oxidation state.

\section{References and Notes}

1. C. K. Gupta and N. Krishnamurthy, Int. Mater. Rev. 37, 197 (1992).

2. K. L. Nash, in Lanthanides/Actinides: Chemistry, vol. 18 of Handbook on the Physics and Chemistry of Rare Earths, K. A. Gschneidner Jr. et al., Eds. (Elsevier Science, Amsterdam, 1994), pp. 197-238.

3. J. Kaczmark, in Industrial Applications of Rare Earth Elements, K. A. Gschneidner Jr., Ed. (American Chemical Society, Washington, DC, 1981), pp. 135166.

4. G. Adachi et al., J. Less Common Met. 169, L1 (1991).

5. K. Murase et al., J. Alloys Compounds 198, 31 (1993).

6. Bull. Chem. Soc. Jpn. 65, 2724 (1992).

7. D. A. Johnson, Adv. Inorg. Chem. Radiochem. 20, 1 (1977).

8. C. Meyer, Chem. Rev. 88, 93 (1988).

9. G. Czack et al., Gmelin Handbook of Inorganic Chemistry Sc, Y, La-Lu Rare Earth Elements Part C4b (Springer-Verlag, Berlin, ed. 8, 1982).
10. I. Barin, Thermodynamical Data of Pure Substances ( $\mathrm{VCH}$, Weinheim, Germany, ed. 3, 1995).

11. L. S. Kudin et al., Electrochem. Soc. Proc. 97-39, 704 (1997).

12. A. V. Hariharan and H. A. Eick, High Temp. Sci. 4, 91 (1972).

13. T. Uda, T. H. Okabe, Y. Waseda, K. T. Jacob, J. Alloys Compounds 284, 282 (1999).

14. T. Uda, T. H. Okabe, Y. Waseda, J. Jpn. Inst. Met. 62 , 796 (1998).

15. G. I. Novikov and O. G. Polyachenok, Russ. Chem. Rev. 33, 343 (1964).

16. D. A. Johnson, J. Chem. Soc. A XX, 2578 (1969).
17. G. Adachi, Ed., Science of Rare Earths (Kagakudojin, Kyoto, Japan, 1999), pp. 188-194.

18. J. Shibata and S. Matsumoto, Mater. Sci. Forum 315317, 268 (1999).

19. H. Bergmann, Ed., Gmelin Handbook of Inorganic Chemistry Sc, Y, La-Lu Rare Earth Elements Part C6 (Springer-Verlag, Berlin, ed. 8, 1978).

20. L. R. Morss and T. G. Spence, Z. Anorg. Allg. Chem. 616, 162 (1992).

21. We are grateful to T. H. Okabe and Y. Waseda of Tohoku University and K. Murase of Kyoto University for support and useful discussions.

20 June 2000; accepted 7 August 2000

\title{
A Mechanical Model for Intraplate Earthquakes: Application to the New Madrid Seismic Zone
}

\begin{abstract}
Shelley J. Kenner*† and Paul Segall
We present a time-dependent model for the generation of repeated intraplate earthquakes that incorporates a weak lower crustal zone within an elastic lithosphere. Relaxation of this weak zone after tectonic perturbations transfers stress to the overlying crust, generating a sequence of earthquakes that continues until the zone fully relaxes. Simulations predict large (5 to 10 meters) slip events with recurrence intervals of 250 to 4000 years and cumulative offsets of about 100 meters, depending on material parameters and far-field stress magnitude. Most are consistent with earthquake magnitude, coseismic slip, recurrence intervals, cumulative offset, and surface deformation rates in the New Madrid Seismic Zone. Computed interseismic strain rates may not be detectable with available geodetic data, implying that low observed rates of strain accumulation cannot be used to rule out future damaging earthquakes.
\end{abstract}

During the winter of 1811 to 1812 , three large intraplate earthquakes with cumulative magnitude $M_{\mathrm{w}}=7.5$ to 8.3 (1) occurred within the New Madrid Seismic Zone (NMSZ) (Fig. 1). Despite extensive research, our understanding of the processes that generate intraplate earthquakes such as these is limited. Seismicity in the NMSZ is presently limited to a zone $\sim 250 \mathrm{~km}$ long (Fig. 1). Events on the two northeast trending lineaments have right-lateral mechanisms, whereas events in the northwest trending, compressional stepover are predominately reverse (2).

Dating of paleoliquefaction features and trenching along the central thrust fault reveal at least two prehistoric earthquakes of comparable size to the 1811-12 earthquakes, with interevent times of $\sim 500$ years (3). Fault slip during the 1811-12 earthquakes is estimated at $\sim 8 \mathrm{~m}$, based on isoseismal areas and empirical mag-

Department of Geophysics, Stanford University, Stanford, CA 94305, USA.

*To whom correspondence should be addressed. Email: kenner@gps.caltech.edu

$\dagger$ Present address: Division of Geological and Planetary Sciences, Seismological Laboratory, California Institute of Technology, Mail Code 252-21, Pasadena, CA 91125, USA. nitude-length relations (4). Gravity and magnetic studies require strike-slip offsets of $<10$ $\mathrm{km}$ (5). However, dip-slip offsets of $<70$ to $80 \mathrm{~m}$ on the central thrust $(6,7)$ limit cumulative strike-slip displacements to $\sim 100 \mathrm{~m}$, implying a recent initiation of faulting (4). Sliprates on the central thrust fault increased from $<0.001 \mathrm{~mm} /$ year before the Holocene to 1.8 $\mathrm{mm} /$ year during the Holocene and $6.2 \mathrm{~mm} /$ year in the Late Holocene (7).

Comparison of Global Positioning System (GPS) data with 1950s triangulation surveys indicated strain accumulation rates of $1.1 \times$ $10^{-7}$ year $^{-1}\left(0.44 \times 10^{-7}\right.$ to $2.0 \times 10^{-7}$ at $95 \%$ confidence) in the NMSZ (8). GPS surveys conducted between 1991 and 1997, however, yield strain accumulation rates that are not significantly different from zero ( 9 , 10 ), given the current $95 \%$ confidence detection level of $\sim 1 \times 10^{-7}$ year $^{-1}$ (11). GPS site velocities far from the seismic zone are negligible (9), consistent with the intraplate tectonic environment. The low strain-rates led Newman et al. to conclude that the potential for large earthquakes in the NMSZ was "significantly overestimated" (9). These authors interpret the geodetic velocities with an infinitely long screw dislocation (12) in 